\title{
Real-World Direct Health Care Costs Associated with Psychotropic Polypharmacy Among Adults with Common Cancer Types in the United States
}

\author{
Ami M. Vyas, PhD, MS, MBA; Stephen J. Kogut, PhD, MBA, RPh; and Hilary Aroke, PhD, MD, MPH
}

\begin{abstract}
BACKGROUND: Psychotropic polypharmacy is not uncommon among cancer patients and may contribute to the increased direct health care cost burden in this population.

OBJECTIVE: To estimate average direct health care costs in the year following cancer diagnosis among cancer patients receiving psychotropic polypharmacy compared with those without psychotropic polypharmacy, using a multivariable analysis framework.

METHODS: A retrospective cross-sectional study was conducted among patients aged 18 years and older diagnosed with the most commonly occurring cancers (breast, prostate, lung, and colorectal) in the United States during 2011-2012 using the deidentified Optum Clinformatics Data Mart commercial claims database. Psychotropic polypharmacy was defined as concurrent use of 2 or more psychotropic medications for at least 90 days. Direct health care costs in the year following cancer diagnosis were estimated as total medical payments made by the health plans and were derived from claims files. A generalized linear regression model with loglink function and gamma distribution was used to model average direct health care costs, controlling for baseline patient demographic and clinical covariates.
\end{abstract}

RESULTS: Average annual direct health care costs for cancer patients with psychotropic polypharmacy $(\$ 53,497$; SD $\$ 72,590)$ were higher than those without psychotropic polypharmacy $(\$ 38,255$; SD $\$ 59,844)$, with an unadjusted average cost difference of $\$ 15,242(P<0.0001)$. In the adjusted regression model, the average difference in costs shrunk to $\$ 5,888$ but remained notable. When examined by type of cancer, average direct health care costs for all cancer patients with psychotropic polypharmacy were significantly higher than those for patients without psychotropic polypharmacy, except for colorectal cancer patients.

CONCLUSIONS: Overall health care costs were higher among cancer patients with psychotropic polypharmacy compared with those without psychotropic polypharmacy. Our findings support the need for future research to better understand the benefits and risks of psychotropic polypharmacy, given its potential to cause adverse health outcomes and avoidable health care utilization and costs for this vulnerable patient population.

J Manag Care Spec Pharm. 2019;25(5):555-65

Copyright @2019, Academy of Managed Care Pharmacy. All rights reserved.

\section{What is already known about this subject}

Psychotropic medications are frequently prescribed to cancer patients leading to psychotropic polypharmacy, which is highly concerning owing to increased risk of drug-drug interactions. No studies have evaluated the effect of psychotropic polypharmacy on annual direct health care costs among cancer patients using a large commercial claims database.

\section{What this study adds}

A total of $7.4 \%$ of cancer patients received psychotropic polypharmacy in the year following cancer diagnosis.

Compared with cancer patients without psychotropic polypharmacy, those with psychotropic polypharmacy had notably higher direct health care costs $(\$ 5,888)$ and costs by types of specific health services in the year following their cancer diagnosis.

Total health care costs and costs by types of specific health services remained higher for patients with breast cancer, lung cancer, or prostate cancer who were prescribed psychotropic polypharmacy.

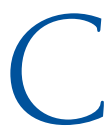
ancer is the second leading cause of death in the United States and the second most costly medical condition..$^{1-3}$ The annual direct costs of cancer care were estimated to increase from $\$ 104$ billion in 2006 to $\$ 174$ billion in 2020.,5 Because cancer and mental health are considered critical priority areas of health services research, comprehensive patientcentered care in oncology has been emphasized in international treatment guidelines and standards and recommendations. ${ }^{6-8}$ Improving quality of cancer care requires recognizing and addressing both physical and mental health of cancer patients during treatment and after care, to augment quality for life of adults with cancer. ${ }^{7,8}$

More than one third of newly diagnosed adults with cancer experience mental health and adjustment disorders. ${ }^{9-11}$ Psychotropic medications are frequently prescribed by oncologists and psychiatrists to the adults with cancer. ${ }^{12-15}$ Psychotropic medication use has been reported in $17 \%$ of the cancer survivors in the United States, with antidepressants and anxiolytics being prescribed to $13.7 \%$ and $5.9 \%$ of adults with cancer, respectively. ${ }^{16}$ Psychotropic medications are commonly 
used in adults with cancer for several nonpsychotropic conditions as well, including fatigue, insomnia, and pain. ${ }^{17-21}$

Since the use of psychotropic medications is frequently indicated in adults with cancer, psychotropic polypharmacy, defined as concurrent use of 2 or more psychotropic medications, ${ }^{22,23}$ is frequently encountered in this vulnerable group. Psychotropic polypharmacy among adults with cancer is of concern due to additive effects and increased risk of drug-drug interactions not only between psychotropic medications but also between psychotropic medications and anticancer medications. ${ }^{24,25}$

Of the studies that have evaluated the use of psychotropic medications in adults with cancer, ${ }^{12-16,20,21,26-29}$ most are outdated ${ }^{20,21,26,27}$; some were conducted in the non-U.S. settings ${ }^{12-15}$; and others have focused mostly on the treatment of depression. ${ }^{30-34}$ One study by Vyas et al. (2018) evaluated psychotropic polypharmacy among adults with cancer and reported higher prevalence and rates of psychotropic polypharmacy that varied by type of cancer. ${ }^{29}$ There is a dearth of studies examining how psychotropic medication use and, in particular, psychotropic polypharmacy among adults with cancer affect direct health care costs, using large U.S.-based databases with wide geographic coverage. Given that health care costs associated with cancer care are substantially increasing and pose a greater economic burden to the United States,$^{35}$ identifying the factors contributing to higher health care costs in this vulnerable population is increasingly essential for health care policymakers, health care systems, physicians, and society as a whole.

To the best of our knowledge, no study has analyzed direct health care costs by psychotropic polypharmacy in adults with cancer. Therefore, the primary objectives of this study were to estimate the average direct health care costs in the year following cancer diagnosis among adults with cancer with and without psychotropic polypharmacy and to estimate average costs by types of specific health care services, after adjusting for patient baseline characteristics and clinical factors. Understanding if psychotropic polypharmacy signifies greater cost burden among adults with cancer may help in identifying the sources of higher costs and target opportunities to reduce adverse outcomes and address unnecessary use and costs of health care services.

\section{Methods}

\section{Study Design and Data Source}

This retrospective observational cross-sectional study was conducted to assess how psychotropic polypharmacy affects total direct health care costs during the first year following cancer diagnosis among adults with cancer. This study used the Optum Clinformatics Data Mart administrative claims database. This database provides claims for medical services, prescription services, laboratory results, and hospital services for more than 23 million unique privately insured patients and Medicare Advantage enrollees from geographically diverse regions across the country during 2010-2013. Diagnoses information on the claims is recorded using International Classification of Disease, Ninth Revision, Clinical Modification (ICD-9-CM) codes. Health care procedures are recorded using Current Procedural Terminology, 4th Edition and the Healthcare Common Procedure Coding System. Pharmacy claims data include information on drug name, drug strength, dosage form, fill date, days of supply, American Hospital Formulary Service (AHFS) Pharmacologic Therapeutic Classification System codes, National Drug Code (NDC) numbers, and cost data. The enrollment files provide information on demographic and enrollment status. Since no directly identifiable protected health information was used in this study, it was considered exempt by the University of Rhode Island Institutional Review Board.

\section{Study Cohort}

The study cohort consisted of patients aged $\geq 18$ years, who were diagnosed with cancer between January 1, 2011, and December 31, 2012. We limited this study to female breast, prostate, colorectal, and lung cancers, which are the most common cancers among U.S. adults. ${ }^{36}$ Adults with cancer were required to have complete medical coverage and pharmacy benefits, and continuous enrollment in a U.S. health plan for 12 months before and at least 12 months following their cancer diagnosis with medical and prescription coverage. The service date of the first medical claim with a cancer diagnosis was considered as an index date. Adults with cancer were required to have a non-rule-out cancer diagnosis, defined as $\geq 2$ separate medical claims with a cancer diagnosis, with service dates $\geq 30$ days apart. Cancer diagnosis codes could occur in a primary or secondary position on the claim and included ICD-9-CM codes of 174.xx, 175.xx, 233.0x for breast, 185.xx, 233.4x for prostate, 153.xx, 154.xx, 230.3x, 230.4x for colorectal, and 162.xx, 231.2x for lung cancers. ${ }^{37}$ Adults with cancer were also required to have no medical claim for cancer during the year before diagnosis and no claim for cancer metastases (ICD9-CM codes 196.xx to 198.xx) during the year following the index date. Female patients with claims for prostate cancer and male patients with claims for breast cancer were also excluded.

\section{Study Measures}

Dependent Variable: Health Care Costs. Total all-cause health care costs in the year following the index date were defined and computed as the sum of reimbursed costs paid by the health plan for all the medical services and pharmacy services derived from the inpatient and outpatient medical services and outpatient pharmacy services claims files. Average health care costs for specific services (inpatient, outpatient, office visits, emergency department visits, prescription, and other medical services) were estimated and categorized by use of psychotropic polypharmacy during follow-up. 


\section{FIGURE 1 Flowchart for Study Cohort Selection}

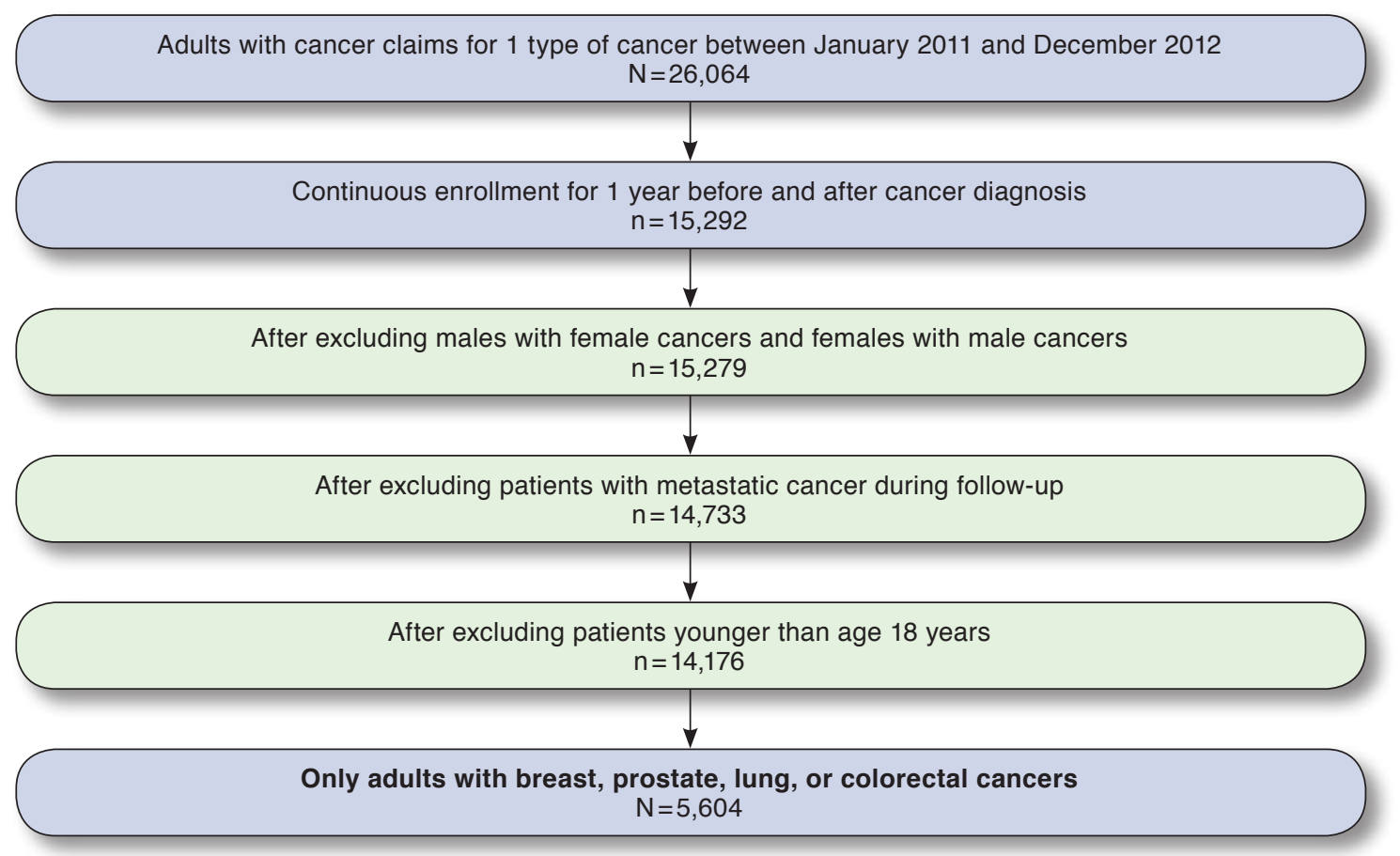

Key Independent Variable: Psychotropic Polypharmacy. Psychotropic medication use was identified from the outpatient pharmacy claims files by using NDC numbers and dispense dates within 1 year following the index date. Psychotropic medications were grouped into major classes according to the AHFS Pharmacologic Therapeutic Classification System: antidepressants, antipsychotics, anticonvulsants, anxiolytics-sedatives-hypnotics (including barbiturates), and central nervous system (CNS) stimulants. Psychotropic polypharmacy was defined as concurrent use of 2 or more psychotropic medications from any of these classes for at least 90 days. ${ }^{22,23}$

Other Independent Variables. Patient demographics comprised of age at cancer diagnosis (18-44 years, 45-54 years, 55-64 years, 65 years and older); gender (male, female); type of health plan (point-of-service, exclusive provider organization, health maintenance organization, and others); and geographic census region in the United States (Northeast, South, Midwest, West). Clinical characteristics comprised baseline comorbidity; mental health conditions; type of primary cancer diagnosis (breast, prostate, lung, colorectal); and use of chemotherapy and radiation therapy during the follow-up period. Charlson Comorbidity Index (CCI) was calculated for each patient based on the presence of diagnosis codes for medical claims during the baseline period. ${ }^{38}$ The calculation of CCI excluded the primary diagnosis of interest (i.e., cancer). Mental health conditions commonly captured in the baseline period were identified from the published literature and included presence of depression, anxiety, dysthymia, post-traumatic stress disorder, bipolar disorder, personality disorders, schizophrenia, and other psychotic disorders, obtained from the medical claims using ICD-9-CM codes for those conditions. ${ }^{39-41}$ Chemotherapy and radiation therapy were identified from the medical claims using ICD-9-CM codes V58.11 and V58.0, respectively.

\section{Statistical Analyses}

Descriptive statistics were used to describe the characteristics of the study cohort. Chi-square tests were used to describe the significant differences between those with and without psychotropic polypharmacy. Average health care costs and costs for specific health care services were statistically compared using t-tests and ratio of means for those with and without psychotropic polypharmacy. A modified Park test was conducted to determine the most appropriate regression model to fit the cost distribution. ${ }^{42}$ Per the findings from the Park test, generalized linear model regression with log-link function and gamma distribution was conducted to evaluate if psychotropic polypharmacy was associated with health care costs, after controlling for baseline covariates. The regression estimates were exponentiated to yield average costs. Significant findings with $P$ values less than or equal to 0.05 levels were discussed. All 
Real-World Direct Health Care Costs Associated with Psychotropic

Polypharmacy Among Adults with Common Cancer Types in the United States

\begin{tabular}{|c|c|c|c|c|c|c|c|}
\hline \multirow[b]{2}{*}{ Variables } & \multicolumn{2}{|c|}{ Total Cohort } & \multicolumn{2}{|c|}{ Psychotropic Polypharmacy } & \multicolumn{2}{|c|}{ No Psychotropic Polypharmacy } & \multirow[b]{2}{*}{$P$ Value } \\
\hline & $\mathrm{N}=5,604$ & $\%$ & $\mathrm{n}=416(7.42 \%)$ & $\%$ & $\mathrm{n}=5,188(92.58 \%)$ & $\%$ & \\
\hline \multicolumn{7}{|l|}{ Age (years) } & 0.0202 \\
\hline $18-44$ & 408 & 7.3 & 26 & 6.4 & 382 & 93.6 & \\
\hline $45-54$ & 1,505 & 26.9 & 109 & 7.2 & 1,396 & 92.8 & \\
\hline $55-64$ & 2,822 & 50.3 & 235 & 8.3 & 2,587 & 91.7 & \\
\hline $65+$ & 869 & 15.5 & 46 & 5.3 & 823 & 94.7 & \\
\hline \multicolumn{7}{|l|}{ Gender } & $<0.0001$ \\
\hline Female & 3,418 & 61.0 & 338 & 9.9 & 3,080 & 90.1 & \\
\hline Male & 2,186 & 39.0 & 78 & 3.6 & 2,108 & 96.4 & \\
\hline \multicolumn{7}{|c|}{ Geographic region } & 0.0500 \\
\hline Midwest & 1,582 & 28.2 & 116 & 7.3 & 1,466 & 92.7 & \\
\hline Northeast & 1,042 & 18.6 & 64 & 6.1 & 978 & 93.9 & \\
\hline South & 2,265 & 40.4 & 192 & 8.5 & 2,073 & 91.5 & \\
\hline West & 714 & 12.8 & 44 & 6.2 & 670 & 93.8 & \\
\hline \multicolumn{7}{|c|}{ Type of insurance plan } & 0.0748 \\
\hline EPO & 727 & 13.0 & 48 & 6.6 & 679 & 93.4 & \\
\hline $\mathrm{HMO}$ & 295 & 5.3 & 18 & 6.1 & 277 & 93.9 & \\
\hline POS & 4,181 & 74.6 & 308 & 7.4 & 3,873 & 92.6 & \\
\hline Other & 401 & 7.2 & 42 & 10.5 & 359 & 89.5 & \\
\hline \multicolumn{7}{|c|}{ Type of cancer } & $<0.0001$ \\
\hline Breast & 2,946 & 52.6 & 271 & 9.2 & 2,675 & 90.8 & \\
\hline Colorectal & 636 & 11.4 & 51 & 8.0 & 585 & 92.0 & \\
\hline Lung & 306 & 5.4 & 44 & 14.4 & 262 & 85.6 & \\
\hline Prostate & 1,716 & 30.6 & 50 & 2.9 & 1,666 & 97.1 & \\
\hline \multicolumn{7}{|c|}{ Number of mental conditions } & $<0.0001$ \\
\hline 0 & 5,256 & 93.8 & 289 & 5.5 & 4,967 & 94.5 & \\
\hline 1 & 288 & 5.1 & 99 & 34.4 & 189 & 65.6 & \\
\hline $2+$ & 60 & 1.1 & 28 & 46.7 & 32 & 53.3 & \\
\hline \multicolumn{7}{|c|}{ Comorbidity score } & $<0.0001$ \\
\hline 0 & 3,729 & 66.5 & 216 & 5.8 & 3,513 & 94.2 & \\
\hline 1 & 1,252 & 22.4 & 123 & 9.8 & 1,129 & 90.2 & \\
\hline $2+$ & 623 & 11.1 & 77 & 12.4 & 546 & 87.6 & \\
\hline
\end{tabular}

statistical analyses were performed using statistical analysis systems software SAS version 9.4 (SAS Institute, Cary, NC).

\section{Results}

\section{Descriptive Characteristics}

Of 5,604 patients included in the study cohort (Figure 1), $52.6 \%$ had breast cancer, $30.6 \%$ had prostate cancer, $11.4 \%$ had colorectal cancer, and 5.4\% had lung cancer (Table 1). A majority of the study cohort was younger than age 65 years (84\%), were females (61.0\%), resided in the South region (40.4\%), and had point-of-service health plans (74.6\%). However, most of these adults with cancer did not have any coded diagnosis for a mental health condition (93.8\%) or a comorbid physical condition $(66.5 \%)$ in the year before their cancer diagnosis.

A total of $7.4 \%$ of adults with cancer were prescribed psychotropic polypharmacy in the year following cancer diagnosis, while $92.6 \%$ had no psychotropic polypharmacy. The characteristics of adults with cancer were significantly different between those with and without psychotropic polypharmacy, except for their type of health insurance plan. Compared with males with cancer, females with cancer were more likely to be prescribed psychotropic polypharmacy $(P<0.0001)$. Adults with cancer in the age group 55-64 years were more likely to be prescribed psychotropic polypharmacy compared with those in other age groups $(P=0.0202)$. Also, those with breast or lung cancer were more likely to be prescribed psychotropic polypharmacy, compared with those with prostate cancer $(P<0.0001)$. In addition, adults with cancer with 2 or more psychiatric conditions $(P<0.0001)$ and physical conditions $(P<0.0001)$ were more likely to be prescribed psychotropic polypharmacy compared with their respective counterparts without psychotropic polypharmacy. 


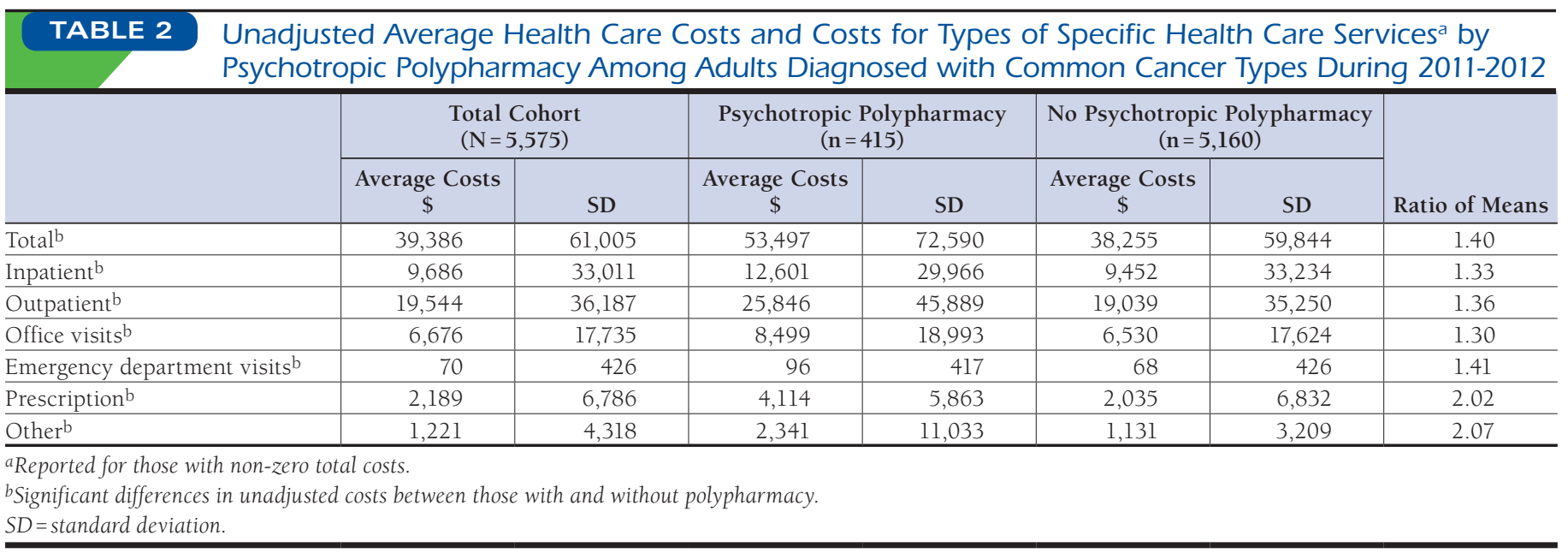

\section{Average Health Care Costs by Psychotropic Polypharmacy}

The average health care costs for the overall study population during the year following cancer diagnosis was $\$ 39,386$ (standard deviation [SD] \$61,005; Table 2). Adults with cancer prescribed psychotropic polypharmacy had average health care costs of $\$ 53,497$ (SD $\$ 72,590$ ) compared with $\$ 38,255$ (SD \$59,844) for those without psychotropic polypharmacy $(P<0.0001)$; an unadjusted average cost difference of $\$ 15,242$ $(P<0.0001)$. Average inpatient, outpatient, office visit, emergency department visit, prescription, and average costs for other medical services were significantly higher for those with psychotropic polypharmacy compared with those without psychotropic polypharmacy ( $\$ 12,601$ vs. $\$ 9,452 ; \$ 25,846$ vs. $\$ 19,039 ; \$ 8,499$ vs. $\$ 6,530 ; \$ 96$ vs. $\$ 68 ; \$ 4,114$ vs. $\$ 2,035$; $\$ 2,341$ vs. $\$ 1,131$, respectively), with ratio of mean costs of 1.33 for inpatient services, 1.36 for outpatient services, and 2.02 for prescription medications.

\section{Average Health Care Costs by Type of Cancer}

Figure 2 shows average health care costs and costs by types of specific services stratified by use of psychotropic polypharmacy for each cancer type. Average health care costs during the year following cancer diagnosis for all adults with cancer with psychotropic polypharmacy were significantly higher than those without psychotropic polypharmacy, except for colorectal cancer patients $(\$ 60,182$ vs. $\$ 74,150)$. When costs were examined by types of specific health care services, inpatient costs, and outpatient costs for patients with breast, prostate, and lung cancer with psychotropic polypharmacy were higher than for their counterparts without psychotropic polypharmacy. However, these costs were lower for colorectal cancer patients with psychotropic polypharmacy compared with those without psychotropic polypharmacy. Costs associated with office visits for patients with breast cancer or lung cancer with psychotropic polypharmacy were higher than for their counterparts without psychotropic polypharmacy, whereas these costs were lower for patients with prostate cancer or colorectal cancer with psychotropic polypharmacy compared with their counterparts without psychotropic polypharmacy. Costs for emergency services and prescription medications for adults with breast, prostate, and colorectal cancer with psychotropic polypharmacy were higher than for their counterparts without psychotropic polypharmacy. On the other hand, these costs were lower for adults with lung cancer with psychotropic polypharmacy compared with those without psychotropic polypharmacy.

\section{Predictors of Health Care Costs by Presence of Psychotropic Polypharmacy}

Compared with adults with cancer who had no psychotropic polypharmacy, average health care costs in the year following cancer diagnosis remained significantly higher for those with psychotropic polypharmacy by $\$ 5,888(22.6 \%)$ in a multivariable gamma regression (Table 3). Adults with cancer in age groups 18-44 years, 45-54 years, and 55-64 years had overall adjusted average costs that were $75.0 \%(P<0.0001), 27.9 \%$ $(P<0.0001)$, and $13.6 \%(P=0.0107)$ higher, respectively, than those aged 65 years and older. Compared with females with cancer, males with cancer had significantly higher adjusted average costs in the year following cancer diagnosis $(P=0.015)$. Adults with cancer who resided in South or West regions of the country had overall adjusted costs that were higher by $17.0 \%$ and $38.8 \%$, respectively, than those who resided in the Midwest.

Adults with colorectal cancer or lung cancer had average health care costs that were $89.0 \%(P<0.0001)$ and $61.5 \%$ $(P<0.0001)$ higher than those who had breast cancer. In addition, adults with cancer who had comorbidity score of at least 1 had significantly higher average health care costs than those with no comorbid physical conditions $(P<0.0001)$. Compared 


\section{FIGURE 2 Unadjusted Average Health Care Costs by Type of Cancer Among Adults with and Without} Psychotropic Polypharmacy

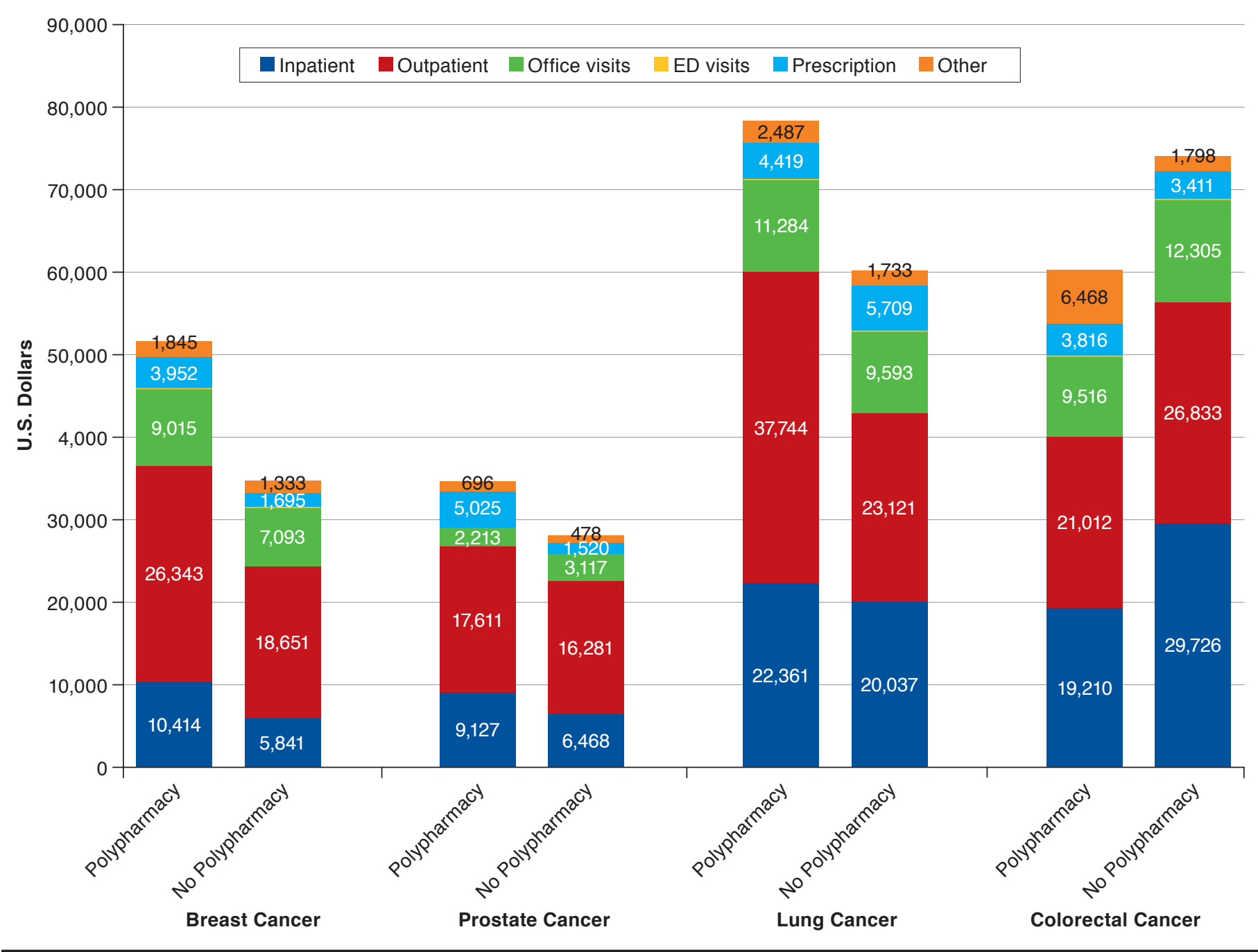

$E D=$ emergency department.

with the adults with cancer with zero comorbidity score, the overall adjusted costs were higher by $20.2 \%$ for those with comorbidity score of 1 and by $69.5 \%$ for those with comorbidity score of at least 2 .

\section{Discussion}

This study is the first to use data from a large U.S. health care plan with a wide geographic coverage to examine average overall direct health care costs, according to service types among adults with cancer prescribed psychotropic polypharmacy. We found that average health care costs were significantly higher among adults with cancer with psychotropic polypharmacy compared with those without psychotropic polypharmacy. In the adjusted analyses, the presence of psychotropic polypharmacy was significantly associated with higher direct health care costs in this population. Average health care costs by types of specific services also remained higher for those with psychotropic polypharmacy compared with those without psychotropic polypharmacy. As a readily available marker, the use of 2 or more psychotropic medications may identify a patient as more likely to utilize additional health care services, across both the mental health and oncology realms, leading to increased health care costs. Our findings indicate the importance of appropriate management of multiple psychotropic medication use in cancer patients and a close surveillance throughout their cancer care to identify and 
Real-World Direct Health Care Costs Associated with Psychotropic

Polypharmacy Among Adults with Common Cancer Types in the United States

TABLE 3 Factors Associated with Average Direct Health Care Costs Using Generalized Linear Regression

\begin{tabular}{|c|c|c|c|c|c|}
\hline Variables & Beta & SE & $P$ Value & $\begin{array}{c}\text { Average Adjusted Costs } \\
\$\end{array}$ & $\begin{array}{c}\text { Higher/Lower Costs } \\
\%\end{array}$ \\
\hline Intercept & 10.1698 & 0.1143 & $<0.0001$ & 26,103 & \\
\hline \multicolumn{6}{|c|}{ Psychotropic polypharmacy } \\
\hline Yes & 0.2034 & 0.0667 & 0.0023 & 31,991 & 22.6 \\
\hline \multicolumn{6}{|l|}{ No (ref) } \\
\hline \multicolumn{6}{|l|}{ Age (years) } \\
\hline $18-44$ & 0.5596 & 0.0795 & $<0.0001$ & 45,679 & 75.0 \\
\hline $45-54$ & 0.2463 & 0.0575 & $<0.0001$ & 33,393 & 27.9 \\
\hline $55-64$ & 0.1272 & 0.0498 & 0.0107 & 29,644 & 13.6 \\
\hline \multicolumn{6}{|l|}{$65+$ (ref) } \\
\hline \multicolumn{6}{|l|}{ Gender } \\
\hline \multicolumn{6}{|l|}{ Female (ref) } \\
\hline Male & 0.1978 & 0.0818 & 0.015 & 31,812 & 21.9 \\
\hline \multicolumn{6}{|c|}{ Geographic region } \\
\hline \multicolumn{6}{|c|}{ Midwest (ref) } \\
\hline Northeast & 0.0848 & 0.0505 & 0.0933 & 28,413 & 8.8 \\
\hline South & 0.1588 & 0.0417 & 0.0001 & 30,595 & 17.2 \\
\hline West & 0.3277 & 0.0563 & $<0.0001$ & 36,225 & 38.8 \\
\hline \multicolumn{6}{|c|}{ Type of insurance plan } \\
\hline EPO & 0.1555 & 0.0864 & 0.0719 & 30,494 & 16.8 \\
\hline \multicolumn{6}{|l|}{ HMO (ref) } \\
\hline POS & 0.1247 & 0.0752 & 0.0973 & 29,570 & 13.3 \\
\hline Other & 0.2124 & 0.0963 & 0.0274 & 32,280 & 23.7 \\
\hline \multicolumn{6}{|c|}{ Type of cancer } \\
\hline \multicolumn{6}{|c|}{ Breast (ref) } \\
\hline Colorectal & 0.6368 & 0.069 & $<0.0001$ & 49,345 & 89.0 \\
\hline Lung & 0.4794 & 0.086 & $<0.0001$ & 42,159 & 61.5 \\
\hline Prostate & -0.0894 & 0.091 & 0.3257 & 23,871 & -8.6 \\
\hline \multicolumn{6}{|c|}{ Chemotherapy } \\
\hline Yes & 1.3086 & 0.0596 & $<0.0001$ & 96,606 & 270.1 \\
\hline \multicolumn{6}{|c|}{ No (ref) } \\
\hline \multicolumn{6}{|c|}{ Radiation therapy } \\
\hline Yes & 0.8516 & 0.0657 & $<0.0001$ & 61,169 & 134.3 \\
\hline \multicolumn{6}{|c|}{\begin{tabular}{l|l} 
No $(\mathrm{ref})$ \\
\end{tabular}} \\
\hline \multicolumn{6}{|c|}{ Number of mental conditions } \\
\hline \multicolumn{6}{|c|}{\begin{tabular}{l|l}
0 (ref) \\
\end{tabular}} \\
\hline 1 & 0.1467 & 0.0778 & 0.0595 & 30,227 & 15.8 \\
\hline $2+$ & 0.1954 & 0.1654 & 0.2375 & 31,736 & 21.6 \\
\hline \multicolumn{6}{|c|}{ Comorbidity score } \\
\hline \multicolumn{6}{|c|}{0 (ref) } \\
\hline 1 & 0.1838 & 0.0413 & $<0.0001$ & 31,370 & 20.2 \\
\hline $2+$ & 0.5278 & 0.0568 & $<0.0001$ & 44,250 & 69.5 \\
\hline
\end{tabular}

Note: Negative percentage values indicate lower costs.

$E P O=$ exclusive provider organization; $H M O=$ health maintenance organization; $P O S=$ point of service; ref = reference group; $S E=$ standard error

address clinical events and potential causes that may lead to increased health care costs. This study underscores the need for future research to better understand the benefits and risks of psychotropic polypharmacy, given the potential for psychotropic polypharmacy to cause adverse health outcomes and avoidable health care resource utilization for this vulnerable patient population.
A total of $7.4 \%$ of adults with cancer received psychotropic polypharmacy in the year following cancer diagnosis, and the rates were higher among adults with lung, colorectal and breast cancers compared with those with prostate cancer. Our findings on psychotropic medication use are consistent with previous published studies. A study that used national survey data reported higher use of psychotropic medications in breast, 
colorectal, and lung cancer survivors ${ }^{16}$; a study conducted using data of hospitalized cancer patients from a single cancer center reported that $23 \%$ of these patients received 2 psychotropic medications, while $36 \%$ received 3 or more psychotropic medications. ${ }^{20}$ Another study reported higher use of antidepressants, sedatives, and hypnotics in elderly cancer patients compared with their noncancer counterparts; however, this study did not evaluate psychotropic polypharmacy in these patients. ${ }^{43}$ The findings from our study underscore the need for close monitoring of adverse drug-drug interactions in adults with breast, colorectal, and lung cancer.

A striking result of our study was the fact that the annual adjusted average direct health care costs for adults with cancer with psychotropic polypharmacy were significantly higher than those without psychotropic polypharmacy by an amount of $\$ 5,888$. In addition, the average inpatient, outpatient, office visit, emergency department visit, and prescription costs were also significantly higher among adults with cancer with psychotropic polypharmacy than those without psychotropic polypharmacy. These patients received multiple and concurrent psychotropic medications in addition to their routine anticancer drugs, which ultimately increases their risk of moderate to severe drug-drug interactions, not only between psychotropic medications but also between psychotropic medications and anticancer drugs. ${ }^{24,25,44,45}$ In fact, 1 study identified at least 1 potential drug interaction in $27 \%$ of cancer patients, of which $86 \%$ were of moderate to major severity. ${ }^{46}$ Hence, psychotropic polypharmacy among adults with cancer may cause clinically meaningful drug-drug interactions that result in high risk for adverse events and their management, potentially contributing to higher health care costs in this group. Several published studies and reports have identified that drug-drug interactions with psychotropic medications in the general population or populations with certain other medical conditions (e.g., dementia and schizophrenia) result in increased health care utilization and, hence, costs. ${ }^{47-50}$ The findings from our study emphasize the importance of comprehensive patient-centered care potentially including the use of psychosocial interventions, mindfulness-based cognitive therapies and social support interventions in adults with cancer, and a closer collaboration between oncology and psychiatry services, in order to manage psychotropic polypharmacy and reduce associated health care costs.

When average health care costs in the year following cancer diagnosis were examined by psychotropic polypharmacy for each cancer type, adults with breast cancer, lung cancer, or prostate cancer with psychotropic polypharmacy had higher average health care costs compared with their respective counterparts without psychotropic polypharmacy; the difference was significant for breast cancer or lung cancer cases. Because there are no published studies that report average health care costs by psychotropic polypharmacy for adults with cancer, our study findings could not be compared with those found in the literature. A study by Zuckerman et al. (2014) evaluated use and spending of antidepressants, sedatives, and hypnotics in elderly cancer patients enrolled in Medicare and found that cancer type was not associated with spending on these medications ${ }^{43}$ However, this study did not specifically evaluate concurrent use of antidepressants, sedatives and hypnotics, and, hence, psychotropic polypharmacy and overall health care costs in cancer patients.

The difference in average health care costs due to psychotropic polypharmacy in adults with breast cancer or lung cancer may be partly explained by the findings reported by Yap et al (2010). ${ }^{51}$ In that study, the authors specifically described the number of interactions between anticancer drugs and antidepressants for several types of cancers, reporting a very high number of interactions for breast cancer, followed by lung cancer. Thus, adults with breast cancer or lung cancer prescribed concurrent or multiple antidepressants and other psychotropic medications may be at higher risk of experiencing increased number of drug-drug interactions, which may be one of the causes of higher health care costs.

Another study examined drug-drug interactions in adults with cancer prescribed oral anticancer drugs and reported high prevalence of CNS-related interactions resulting from increased use of CNS depressant medications (e.g., antidepressants and benzodiazepines). ${ }^{52}$ Therefore, adults with cancer prescribed concurrent CNS-depressant medications may be at higher risk of experiencing CNS-related interactions, potentially causing increased health care costs.

Another striking finding of our study was that adults with colorectal cancer with psychotropic polypharmacy had lower average health care costs compared with those without psychotropic polypharmacy. To understand this inconsistent finding, we evaluated costs by gender and by presence of psychotropic polypharmacy and found that females with colorectal cancer with psychotropic polypharmacy had significantly lower average health care costs than those without psychotropic polypharmacy $(\$ 49,620$ vs. $\$ 67,676 ; P<0.05)$. However, in males with colorectal cancer, there was a minimal difference in average health care costs by presence and absence of psychotropic polypharmacy ( $\$ 83,287$ vs. $\$ 79,511)$. Therefore, females with colorectal cancer without psychotropic polypharmacy could be driving overall higher costs in the colorectal cancer population without psychotropic polypharmacy (data not shown in tabular form). Future studies are needed to explore additional reasons for lower health care costs in adults with colorectal cancer on multiple psychotropic medications. 


\section{Limitations}

The following limitations should be considered when interpreting the study findings. Measuring clinical outcomes and adverse events caused by psychotropic polypharmacy were beyond the scope of this study; therefore, the findings should be interpreted with caution. Health care services obtained outside of the health care plan may not be captured, thereby causing underestimation of overall health care costs. Nonmedical care and indirect costs such as costs paid to caregiver, out-ofpocket costs, and productivity losses that were not captured and included in the analyses may also underestimate the true health care costs. Additionally, evaluating cancer-related costs was beyond the scope of this study.

Because data from a narrow time frame were used, costs were not adjusted for medical inflation. Data describing certain characteristics such as race/ethnicity; socioeconomic status; severity of comorbidities; clinical information on cancer (e.g., tumor size, tumor stage, and tumor grade); health status; and patient preferences, which could affect selection of psychotropic medication use and hence health care costs, were not captured and adjusted for in our analysis. Since the data for only 4 years were available for our study, a relatively shorter baseline period to capture mental health comorbidities (i.e., 12 months before cancer diagnosis) were used. Finally, because this study was conducted in a U.S. managed care population using data for adults with cancer diagnosed during 2011-2012, the findings may not be generalizable to other populations.

\section{Conclusions}

In the year following cancer diagnosis, overall annual average health care costs and costs by types of specific services were significantly higher among adults with breast, prostate, or lung cancer with psychotropic polypharmacy compared with those without psychotropic polypharmacy. Our findings support the need to better understand the benefits and risks of psychotropic polypharmacy, given the potential for psychotropic polypharmacy to cause adverse outcomes and avoidable health care utilization for this vulnerable patient population. Future studies aimed at identifying the potential causes of higher costs in cancer patients with psychotropic polypharmacy and also expensive drug-drug interactions involving psychotropic medications and anticancer medications that may lead to adverse clinical outcomes and higher costs in this patient population, are warranted.

\section{Authors}

AMI M. VYAS, PhD, MS, MBA; STEPHEN J. KOGUT, PhD, MBA, RPh; and HILARY AROKE, PhD, MD, MPH, Department of Pharmacy Practice, University of Rhode Island College of Pharmacy, Kingston.

AUTHOR CORRESPONDENCE: Ami M. Vyas, PhD, MS, MBA, Department of Pharmacy Practice, University of Rhode Island College of Pharmacy, 7 Greenhouse Rd., Kingston, RI 02881. Tel.: 401.874.7255; E-mail: avyas@uri.edu.

\section{DISCLOSURES}

This study was funded by the American Association of Colleges of Pharmacy (AACP) New Investigator Award mechanism, which was received by Vyas. Aroke was partially supported by the AACP grant for conducting data analysis of the study. Kogut is partially supported by Institutional Development Award Number U54GM115677 from the National Institute of General Medical Sciences of the National Institutes of Health, which funds Advance Clinical and Translational Research (Advance-CTR). The content is solely the responsibility of the authors and does not necessarily represent the official views of the National Institutes of Health and the AACP. The authors report no conflicts of interest.

An abstract of this study was presented as a poster at the American Association of Colleges of Pharmacy Annual Meeting on July 22, 2018, in Boston, MA.

\section{REFERENCES}

1. Centers for Disease Control and Prevention. United States cancer statistics data visualizations tool. 2017. Available at: https://www.cdc.gov/cancer/dcpc/ data/types.htm. Accessed March 5, 2019.

2. Siegel RL, Miller KD, Jemal A. Cancer statistics, 2017. CA Cancer J Clin. 2017;67(1):7-30

3. Soni A; Medical Expenditure Panel Survey. Top five most costly conditions among adults age 18 and older, 2012: estimates for the U.S. civilian noninstitutionalized population. Statistical brief \#471. April 2015. Available at http://www.meps.ahrq.gov/mepsweb/data_files/publications/st471/stat471. pdf. Accessed March 5, 2019.

4. National Cancer Institute. Online summary of trends in US cancer control measures: cancer trends progress report: 2009/2010 update. February 2019. Available at: http://www.progressreport.cancer.gov. Accessed March 5, 2019. 5. Mariotto AB, Yabroff KR, Shao Y, et al. Projections of the cost of cancer care in the United States: 2010-2020. J Natl Cancer Inst. 2011;103:117-28.

6. National Comprehensive Cancer Network. NCCN clinical practice guidelines in oncology: distress management. Version 2.2019. March 5, 2019. Available at: https://www.nccn.org/professionals/physician_gls/pdf/distress. pdf. Accessed March 5, 2019.

7. Adler NE, Page AEK; Institute of Medicine; Committee on Psychosocial Services to Cancer Patients/Families in a Community Setting, eds. Cancer Care for the Whole Patient: Meeting Psychosocial Health Needs. Washington, DC: National Academies Press; 2008

8. American College of Surgeons Commission on Cancer. Cancer program standards 2012: ensuring patient-centered care. Version 1.2.1. 2012. Available at: https://www.facs.org/ /media/files/quality\%20programs/cancer/ coc/programstandards2012.ashx. Accessed March 5, 2019

9. Singer S, Das-Munshi J, Brahler E. Prevalence of mental health conditions in cancer patients in acute care-a meta-analysis. Ann Oncol. 2010;21:925-30. 
10. Mitchell AJ, Chan M, Bhatti H, et al. Prevalence of depression, anxiety, and adjustment disorder in oncological, haematological, and palliativecare settings: a meta-analysis of 94 interview-based studies. Lancet. 2011;12(2):160-74

11. Mehnert A, Brahler E, Faller H, et al. Four-week prevalence of mental disorders in patients with cancer across major tumor entities. J Clin Oncol. 2014:32(31):3540-46.

12. Desplenter F, Bond C, Watson M, et al. Incidence and drug treatment of emotional distress after cancer diagnosis: a matched primary care casecontrol study. Br J Cancer. 2012;107(9):1644-51.

13. Ng CG, Boks MP, Smeets HM, Zainal NZ, de Wit NJ. Prescription patterns for psychotropic drugs in cancer patients: a large population study in the Netherlands. Psychooncology. 2013;22(4):762-67.

14. de Bock GH, Musters RF, Bos HJ, Schröder CP, Mourits MJ, de Jong-van den Berg LT. Psychotropic medication during endocrine treatment for breast cancer. Support Care Cancer. 2012;20(7):1533-40.

15. Farriols C, Ferrandez O, Planas J, Ortiz P, Mojal S, Ruiz AI. Changes in the prescription of psychotropic drugs in the palliative care of advanced cancer patients over a seven-year period. J Pain Symptom Manage. 2012;43(5):945-52.

16. Punekar RS, Short PF, Moran JR. Use of psychotropic medications by U.S. cancer survivors. Psychooncology. 2012;21(11):1237-43.

17. Lee R, Spencer PSJ. Antidepressants and pain: a review of pharmacological data supporting the use of certain tricyclics in chronic pain. J Int Med Res. 1977;5(1 Suppl):146-56

18. Qu D, Zhang Z, Yu X, Zhao J, Qiu F, Huang J. Psychotropic drugs for the management of cancer-related fatigue: a systematic review and metaanalysis. Eur J Cancer Care (Engl). 2015;25(6);970-79.

19. Thekdi SM, Irarrazaval ME, Dunn LB. Psychopharmacological interventions. In: Grassi L, Riba M, eds. Clinical Psycho-Oncology: An International Perspective. Chichester, UK: Willey-Blackwell; 2012:109-26.

20. Stiefel FC, Kornblith AB, Holland JC. Changes in prescription patterns of psychotropic drugs for cancer patients during a 10-year period. Cancer. 1990;65(4):1048-53

21. Derogatis L, Feldstein M, Morrow G, et al. A survey of psychotropic drug prescriptions in an oncology population. Cancer. 1979;44(5):1919-29.

22. Correll CU, Gallego JA. Antipsychotic polypharmacy: a comprehensive evaluation of relevant correlates of a long-standing clinical practice. Psychiatr Clin North Am. 2012;35(3):661-81.

23. Leckman-Westin E, Kealey E, Gupta N, et al. Validation of a claimsbased antipsychotic polypharmacy measure. Pharmacoepidemiol Drug Saf. 2014;23(6):628-35.

24. Sandson NB, Armstrong SC, Cozza KL. An overview of psychotropic drug-drug interactions. Psychosomatics. 2005;46(5):464-94.

25. Yap KY, Tay WL, Chui WK, Chan A. Clinically relevant drug interactions between anticancer drugs and psychotropic agents. Eur J Cancer Care (Engl). 2011;20(1):6-32

26. Goldberg R, Mor V. A survey of psychotropic use in terminal cancer patients. Psychosomatics. 1985;26(9):745-51.

27. Cullivan R, Crown J, Walsh N. The use of psychotropic medication in patients referred to a psycho-oncology service. Psychooncology. 1998;7(4):301-06

28. Braun IM, Rao SR, Meyer FL, Fedele G. Patterns of psychiatric medication use among nationally representative long-term cancer survivors and controls. Cancer. 2015;121(1):132-38.

29. Vyas A, Kogut S, Aroke H, Buchanan A. Psychotropic polypharmacy during the initial phase of care among cancer patients in the United States. Cancer Res. 2018;78(13 Suppl):Abstract 4224
30. Ashbury FD, Madlensky L, Raich P, et al. Antidepressant prescribing in community cancer care. Support Care Cancer. 2003;11(5):278-85.

31. Suppli NP, Deltour I, Damkjear LH, et al. Factors associated with the prescription of antidepressive medication to breast cancer patients. Acta Oncol. 2011;50(2):243-51

32. Jones SMW, Rosenberg D, Ludman E, Arterburn D. Medical comorbidity and psychotropic medication fills in older adults with breast and prostate cancer. Support Care Cancer. 2015;23(10):3005-09.

33. Ng CG, Boks MPM, Zainal NZ, de Wit NJ. The prevalence and pharmacotherapy of depression in cancer patients. J Affect Disord. 2011;131(1-3):1-7.

34. Janberidze E, Hjermstad MJ, Brunelli C, et al. The use of antidepressants in patients with advanced cancer-results from an international multicenter study. Psychooncology. 2014;23(10):1096-102.

35. Yabroff KR, Lund J, Kepka D, Mariotto A. Economic burden of cancer in the United States: estimates, projections and future research. Cancer Epidemiol Biomarkers Prev. 2011;20(10):2006-14

36. American Cancer Society. Cancer facts and figures, 2017. Available at: https://www.cancer.org/content/dam/cancer-org/research/cancer-factsand-statistics/annual-cancer-facts-and-figures/2017/cancer-facts-and-figures-2017.pdf. Accessed March 5, 2019.

37. Chastek B, Harley C, Kallich J, Newcomer L, Paoli CJ, Teitelbaum AH. Health care costs for patients with cancer at the end of life. J Oncol Pract. 2012;8(6):75s-80s.

38. Klabunde CN Potosky AL, Legler JM, Warren JL. Development of a comorbidity index using physician claims data. J Clin Epidemiology. 2000;53(12):1258-67.

39. Kadan-Lottick NS, Vanderwerker LC, Block SD, Zhang B, Prigerson HG. Psychiatric disorders and mental health service use in patients with advanced cancer: a report from the coping with cancer study. Cancer. 2005;104(12):2872-81.

40. Iglay K, Santorelli ML, Hirshfield KM, et al. Diagnosis and treatment delays among elderly breast cancer patients with pre-existing mental illness. Breast Cancer Res Treat. 2017;166(1):267-75.

41. Braun IM, Rao SR, Meyer FL, Fedele G. Patterns of psychiatric medication use among nationally representative long-term cancer survivors and controls. Cancer. 2015;121(1):132-38.

42. Manning WG, Mullahy J. Estimating log models: to transform or not to transform? J Health Econ. 2001;20(4):461-94.

43. Zuckerman IH, Davidoff AJ, Erten MZ, et al. Use of and spending on supportive care medications among Medicare beneficiaries with cancer. Support Care Cancer. 2014;22(8):2185-95.

44. Desmarais JE, Looper KJ. Interactions between tamoxifen and antidepressants via cytochrome P450 2D6. J Clin Psychiatry. 2009;70(12):1688-97. 45. Chan A, Ng TR, Yap KY. Clinically-relevant anticancer-antidepressant drug interactions. Expert Opin Drug Metab Toxicol. 2012;8(2):173-99.

46. Riechelmann RP, Tannock IF, Wang L, Saad ED, Taback NA, Krzyzanowska MK. Potential drug interactions and duplicate prescriptions among cancer patients. J Natl Cancer Inst. 2007;99(8):592-600.

47. Ereshefsky L, Jhee S, Grothe D. Antidepressant drug-drug interaction profile update. Drugs R D. 2005;6(6):323-36.

48. Mark TL, Joish VN, Hay JW, Sheehan DV, Johnston SS, Cao Z. Antidepressant use in geriatric populations: the burden of side effects and interactions and their impact on adherence and costs. Am J Geriatr Psychiatry. 2011;19(3):211-21.

49. Skoldunger A, Fastbom J, Wimo A, Fratiglioni L, Johnell K. Impact of inappropriate drug use on hospitalizations, mortality, and costs in older persons and persons with dementia: findings from the SNAC study. Drugs Aging. 2015;32(8):671-78. 
50. Sengul MCB, Karadag F, Sengul C, Karakulah K, Kalkanci O, Herken H. Risk of psychotropic drug interactions in real world settings: a pilot study in patients with schizophrenia and schizoaffective disorder. Bull Clin Psychopharmacol. 2014;24(3):235-47.

51. Yap KY, Ho Y, Chui WK, Chan A. Harnessing the internet cloud for managing drug interactions with chemotherapy regiments in patients with cancer suffering from depression. Acta Oncologica. 2010;49(8):1235-45.
52. van Leeuwen RW, Brundel DH, Neef C, et al. Prevalence of potential drug-drug interactions in cancer patients treated with oral anticancer drugs. Br J Cancer. 2013;108(5):1071-78. 